\title{
La inteligencia emocional en estudiantes universitarios.
}

Emotional Intelligence in University Students.

\author{
Barrera-Gálvez, $R^{a}$, Solano-Pérez, $C T^{b}$, Arias-Rico, $J^{c}$, Jaramillo-Morales, $O A^{d}$, Jiménez-
} Sánchez, $R C^{e}$

\begin{abstract}
:
Studying at the university level in the modern world is not as easy as it seems. The challenges, the tensions of modern life, the continuous globalization of knowledge in the individual, student, professional, labor field, the pressure of the clock, are situations that respond to an alter the emotional state of the majority of students, taking them on the edge of their own physical and psychic limits. Objective.- To determine the level of emotional intelligence in nursing students of the Autonomous University of the State of Hidalgo, July-December semester of 2018. Methodology.- A descriptive study is carried out with a population of 316 nursing students during the semester of July -December 2018, those who agreed to collaborate with the research, however, obtained reliable results from 265 surveys. Results.- Of the 265 participants, corresponds to the female gender 214 and to the male gender 51 people. The surveys counted as 134 elements grouped into five factors that integrate emotional intelligence; From this, the factor III Emotional advantage, is the aspect of high score. Conclusion.- In the present study it is concluded that most of the students of the Nursing Degree of the Institute of Health Sciences present a level of Emotional Intelligence Media, which favors that students achieve a fuller personality, a To be a competent and quality professional.
\end{abstract}

Keywords:

Emotional intelligence, university, Nursing and emotional intelligence, emotions and health

\section{Resumen:}

Cursar el nivel universitario en el mundo moderno, no es tan fácil como parece. Los retos, las tensiones de la vida moderna, la continua globalización de los conocimientos en el terreno individual, estudiantil, profesional, laboral, la presión del reloj, son situaciones que tienden a alterar el estado emocional de la mayoría de los estudiantes, llevándolos al borde de sus propios límites físicos y psíquicos. Objetivo.- Determinar el nivel de inteligencia emocional en estudiantes de enfermería de la Universidad Autónoma del Estado de Hidalgo, del semestre julio-diciembre del año 2018. Metodología.- Se realizó un estudio descriptivo con una población de 316 estudiantes de enfermería durante el semestre Julio-Diciembre 2018, quienes aceptaron colaborar con la investigación, sin embargo, se obtuvieron resultados confiables de 265 encuestas. Resultados.- De los 265 participantes, corresponden al género femenino 214 y al género masculino 51 personas. Las encuestas contaron como 134 items agrupados en cinco factores que integran la inteligencia emocional; de esto, el factor III Aprovechamiento emocional, es el que apareción con la más alta puntuación. Conclusión.- En el presente estudio se concluye que la mayoría de los estudiantes de la Licenciatura de Enfermería del Instituto de Ciencias de la Salud, presentan un nivel de Inteligencia Emocional Media, que favorece con ello que los estudiantes logren una personalidad más plena, una percepción más satisfactoria de la vida, y como estudiante, además de contar con una gran posibilidad de poder desarrollar sus potencialidades para llegar a ser un profesional competente y de calidad.

\section{Palabras Clave:}

Inteligencia emocional, universitarios, Enfermería e inteligencia emocional, emociones y salud

\footnotetext{
a Autor de Correspondencia, Universidad Autónoma del Estado de Hidalgo, Instituto de Ciencias de la Salud, ORCID: 0000-0002-19495424, Email: rosario_barrera@uaeh.edu.mx

${ }^{\text {b }}$ Universidad Autónoma del Estado de Hidalgo, Instituto de Ciencias de la Salud, ORCID: https://orcid.org/0000-0003-4648-981X, Email: claudia_solano@uaeh.edu.mx

${ }^{c}$ Universidad Autónoma del Estado de Hidalgo, Instituto de Ciencias de la Salud, ORCID: https://orcid.org/0000-0003-0219-0410, Email: josearias.rico@hotmail.com

${ }^{d}$ Universidad Autónoma del Estado de Hidalgo, Instituto de Ciencias de la Salud, ORCID: https://orcid.org/0000-0002-5325-3760, Email: oajmorales@gmail.com

e Universidad Autónoma del Estado de Hidalgo, Instituto de Ciencias de la Salud, ORCID: https://orcid.org/0000-0001-9264-8514, Email: cristyji@hotmail.com
} 


\section{Introducción}

En el escenario universitario de hoy, donde las realidades y estímulos resultan ser múltiples y complejos son diversos los factores que podrían influir en los resultados académicos del estudiante y por ende en su deserción/permanencia en las universidades.

Las tensiones de la vida moderna, la continua globalización de los conocimientos en el terreno individual, estudiantil, profesional, laboral, la presión del reloj, la exigencia de un constante perfeccionamiento, entre otros factores, son situaciones que tienden a alterar el estado emocional de la mayoría de los estudiantes, llevándolos al borde de sus propios límites físicos y psíquicos [1].

No podemos medir el éxito solamente por la capacidad intelectual, sino también por la capacidad emocional. Cualidades como la capacidad de entender los sentimientos propios, la habilidad de entender los sentimientos de los compañeros y el control de las emociones para lograr un fin, son más importantes a veces en un salón de clases que ser exitoso en todas las materias.

Lo ideal para los seres humanos, es alcanzar el equilibrio entre la capacidad intelectual y emocional. Mayer y Salovey definen a la inteligencia emocional como: "Un conjunto de habilidades que explican las diferencias individuales en el modo de percibir y comprender nuestras emociones. Más formalmente, la inteligencia emocional es la habilidad para percibir, valorar y expresar emociones con exactitud, la habilidad para acceder y /0 generar sentimientos que faciliten el pensamiento, para comprender emociones y razonar emocionalmente, y finalmente la habilidad para regular emociones propias y ajenas" [2].

En los informes de evaluaciones nacionales e internacionales donde los estudiantes peruanos obtuvieron tan bajas calificaciones, se asume como uno de los factores condicionantes de estos resultados a la incapacidad emocional de dichos estudiantes, que las causas más importantes son: la baja autoestima, las condiciones socio económicas de la familia y el clima de aula.

En Estados Unidos Salovey, Stroud, Woolery han demostrado que los alumnos universitarios con más IE informan menor número de síntomas físicos, menos ansiedad social y depresión y mejor autoestima, mayor satisfacción interpersonal, mayor utilización de estrategias de afrontamiento activo para solucionar sus problemas. Además, cuando estos alumnos son expuestos a tareas estresantes de laboratorio, perciben los estresores como menos amenazantes y sus niveles de cortisol y presión sanguínea son más bajos [3].

Paralelamente en Australia Ciarrochi, Deane y Anderson; presentan evidencias de que los estudiantes universitarios con alta IE responden al estrés con menor depresión y desesperanza [4].

Por su parte en España, Fernández investiga acerca de los efectos de una adecuada inteligencia emocional sobre el rendimiento académico siendo estos relacionados de forma directamente proporcional.

Encontrando cuatro áreas fundamentales en las que una falta de IE provoca o facilita la aparición de problemas entre los estudiantes estos problemas serian: déficit en los niveles de bienestar y ajuste psicológico del alumnado, disminución en la cantidad y calidad de las relaciones interpersonales, descenso del rendimiento académico, aparición de conductas destructivas y consumo de sustancias adictivas [5].

Villacorta E. en su tesis: "Inteligencia emocional y rendimiento académico en estudiantes de Medicina Humana de la Universidad Nacional de la Amazonía Peruana." Concluye: El nivel o cociente emocional (CE) o nivel de inteligencia emocional (I-CE) total o global (CET) que predomina en los estudiantes de la Facultad de Medicina Humana de la UNAP es el promedio o adecuado (CEP), seguido por el alto o muy alto (CEA) y finalmente por el bajo (CEB). Los resultados evidencian que en los estudiantes investigados predomina significativamente el nivel de inteligencia emocional promedio o adecuado.

Loly y López desarrollaron una investigación titulada: la autoestima y los valores organizacionales en estudiantes universitarios y estudiantes no universitarios de Lima, reportaron resultados que indican que los estudiantes universitarios le dan mayor importancia a los valores como servicio al cliente, la limpieza y la calidad del producto y menor importancia a la honradez, el respeto a la persona y a la responsabilidad; del mismo modo, los estudiantes no universitarios (egresados, graduados y postgraduados) le otorgan mayor importancia al servicio al cliente, limpieza y confianza y menor importancia a la honradez, puntualidad y responsabilidad.

Esta misma jerarquía se observa cuando se establece la relación con la autoestima, sobre todo, cuando la autoestima es muy baja, baja o intermedia; mientras que 
cuando la autoestima es alta o muy alta, se ubica en primer lugar la limpieza y mejora la puntualidad hasta ubicarse en el cuarto lugar en importancia [6].

Centeno realizó una investigación sobre la influencia del programa "Explorando mis Emociones" en la inteligencia emocional de estudiantes del sexto grado de primaria de un centro educativo en Huancayo, llegando a la conclusión que dicho programa influyó positivamente en los estudiantes, ayudando a desarrollar su inteligencia emocional [7].

Javier L, Iparraguirre M, en la investigación: "Inteligencia emocional en estudiantes de la Facultad de Ciencias de la Salud de la Universidad Peruana Los Andes" en Huancayo-Perú, llegan a concluir que los niveles de inteligencia emocional general es muy baja, con resultados semejantes en sus componentes intrapersonal, interpersonal, adaptabilidad manejo del estrés y estado de ánimo general, siendo las carreras profesionales con los más bajos niveles: Enfermería y Odontología, ubicándose preferentemente en el nivel adecuado las carreras de Farmacia y Bioquímica, Psicología y Obstetricia. No se encontraron diferencias significativas entre varones y mujeres en la inteligencia emocional general [8].

\section{Marco conceptual}

Los estudios de inteligencia usualmente hacen referencia a la mente racional basada en el cálculo del coeficiente intelectual que es considerado como el indicador universal de inteligencia de un individuo, sin embargo, existe otro tipo de inteligencia denominado inteligencia emocional la cual hace referencia a la capacidad que tienen las personas de conocer sus sentimientos y de los demás, así como tener la capacidad de manejarlos.

Según Howard Gardner, el coeficiente intelectual no es el único indicador válido para explicar la capacidad cognitiva de los seres humanos, por ello planteó la "teoría de las inteligencias múltiples (IM) según la cual todos los seres humanos poseemos una serie de capacidades cognitivas relativamente autónomas, cada una de ellas definida como una inteligencia distinta."[9]

Como ya hemos mencionado "el término Inteligencia Emocional (IE) fue introducido por Peter Salovey y John Mayer (1990) y poco a poco fue cobrando fuerza hasta quedar firmemente arraigado en la comunidad científica, abriendo nuevas vías de investigación y modificando de forma importante y definitiva la concepción tradicional sobre la inteligencia" [10]
Sin embargo, uno de los autores más destacados en el estudio de la inteligencia emocional es Daniel Goleman, un psicólogo estadounidense que en el año 1995 publicó el libro "Emotional Intelligence" popularizando así el término inteligencia emocional.

"El concepto básico que Goleman defiende en su libro es la idea de que el Coeficiente Intelectual (CI) no es un predictor válido para la felicidad. Parte de la base de que el hecho de tener un $\mathrm{Cl}$ de 120, 130 ó 140 no implica que vayamos a ser más felices, para eso se necesita otra cosa. No es el concepto de inteligencia que tradicionalmente se ha venido utilizando el elemento a esgrimir, sino un concepto que presenta una mayor complejidad: la inteligencia emocional.".

Desde la perspectiva de Goleman la inteligencia emocional implica un control adecuado de las emociones, la capacidad de ser empático, el autocontrol, ser capaz de emoción es un estado mental poderosamente cargado de sentimiento. Esa definición nos permitió excluir las sensaciones puramente sensoriales, como el cansancio o el hecho de cortarnos un dedo, ya que los sentimientos están ligados a una evaluación, es decir a un pensamiento."[11]

Goleman plantea que "conviene pensar en las emociones en términos de familias o dimensiones, y en considerar a las principales familias-la ira, la tristeza, el miedo, la alegría, el amor, la vergüenza, etcétera-como casos especialmente relevantes de los infinitos matices de nuestra vida emocional. Cada una de estas familias se agrupa en torno a un núcleo fundamental, a partir del cual dinamizan -a modo de olas- todas las otras emociones derivadas de ella."[12]

"La inteligencia emocional (o CE), de acuerdo con Goleman, consiste en las habilidades de autoconocimiento, autoreglamentación o autocontrol, motivación, en la habilidad de entender las señales emotivas y conductuales de los demás (empatía) y en las habilidades interpersonales. [...] A diferencia de la inteligencia cognoscitiva $(\mathrm{Cl})$, que permanece sin cambios importantes a lo largo de la vida, el CE se puede desarrollar y mejorar a lo largo del tiempo con un esfuerzo concertado.

Goleman considera que "la inteligencia, entendida de forma tradicional como la capacidad de resolver problemas abstractos, no es siempre la mejor garantía de éxito en la vida personal y social. Hay otros elementos que la escuela no tiene en cuenta en su currículo y que son de vital importancia para responder de forma positiva a las exigencias que el mundo nos presenta. Ese conjunto de elementos ha sido denominado inteligencia emocional. 
Adicionalmente, es importante comprender la diferencia existente entre emociones y sentimientos.

"Las emociones son reacciones a las informaciones (conocimiento) que recibimos en nuestras relaciones con el entorno. La intensidad de la reacción está en función de las evaluaciones subjetivas que realizamos sobre cómo la información recibida va afectar nuestro bienestar."[13]

"Los sentimientos son el balance consciente de nuestra situación; en este balance intervienen varios factores: nuestro estado físico, la marcha de nuestros deseos y proyectos, e sistema de creencias, nuestras experiencias anteriores, entre otros"[14].

La diferencia entre emoción y sentimiento es "que el sentimiento es más duradero y la emoción puede ser breve y repentina."[15]

El cuestionario de inteligencia emocional el cual construyó Daniel Goleman a través de los diferentes estudios que realizaron los diferentes autores que se han interesado en el tema. La afirmación del cuestionario se clasifica en 5 categorías: autoconciencia, autocontrol, aprovechamiento emocional, empatía y habilidad social.

Cada categoría se operacionaliza de la siguiente forma:

Autoconciencia: Se refiere a averiguar cómo hacemos nuestras evaluaciones y cómo manejamos simultáneamente nuestros papeles de actores y observadores al mismo tiempo, reconocer los propios estados de ánimo, los recursos y las instituciones. Hay que identificar las propias emociones, conocer las propias fortalezas y limitaciones.

Autocontrol: Se refiere a manejar los propios estados de ánimo, impulsos y recursos teniendo en cuenta que las emociones en sí mismas son ni buenas ni malas. Se necesita tomar el mando de nuestros pensamientos, dirigir oportunamente nuestras excitaciones nerviosas, llegar a ser buenos solucionadores de problemas, asumir las responsabilidades del propio desempeño laboral, flexibilidad en el manejo de las situaciones de cambio, sentirse cómodo con la nueva información, ideas o situaciones.

Aprovechamiento emocional: Es la combinación de la automotivación y de la autoestima con la que cuenta un ser humano.

Automotivación es usar nuestro sistema emocional para canalizar todo el sistema y mantenerlo en funcionamiento, es decir, tendencias emocionales que guían o facilitan el cumplimiento de las metas establecidas.

Autoestima es el concepto de cada individuo tiene de sí mismo, puede ser positivo o negativo. Es tener un fuerte sentido del propio valor y capacidad.

Se presenta un esfuerzo por mejorar o alcanzar la excelencia, para esto, contamos con nuestro pensamiento positivo, visualización, respiración abdominal, sentido del humor, actividad física, amigos, familia, colegas, un mentor emocional, ya sea real o ficticio y el propio entorno que nos generé un mejor estado emocional.

Empatía: Se refiere a entender los sentimientos, emociones, necesidades y preocupaciones de los otros, es decir, comprender los intereses de los demás para tenerlos en cuenta.

Habilidad social: Es la relación que se tiene con los demás y el canal de comunicación que tiene como fortaleza un ser humano.

Relación con los demás, implica inducir respuestas deseadas en los otros. Depende de poder idear efectivas tácticas de persuasión, saber escuchar abiertamente al resto y elaborar mensajes convenientes, negociar y resolver los desacuerdos que se presenten en el equipo de trabajo, inspirar y guiar a los individuos del grupo, iniciar o administrar las situaciones nuevas, alimentar y reforzar las relaciones interpersonales, trabajar con otros para alcanzar metas, ser capaz de crear empatía para la consecuencia de metas colectivas.

\section{Objetivo}

Determinar el nivel de inteligencia emocional en estudiantes de enfermería de la Universidad Autónoma del Estado de Hidalgo, del semestre julio-diciembre del año 2018.

\section{Metodología}

Se realizó un estudio descriptivo con una población de 316 estudiantes de enfermería durante el semestre JulioDiciembre 2018 en el Instituto de Ciencias de la Salud de la UAEH, que aceptaron colaborar con la investigación. 


\section{Resultados}

Los participantes en la muestra del estudio fueron seleccionados toda vez que cumplieron con los criterios de inclusión, exclusión y/o eliminación; se obtuvieron resultados confiables de 265 encuestas, de una muestra de 316 participantes que se tenían contemplada, por lo que al respecto se cumplió con el cometido de evitar un error de selección y con la muestra del 95\% de confiabilidad con un límite de aceptación del 1.65 con un error aceptable 0.05, mismo que es la diferencia máxima entre la media muestral y la media de la población que se está dispuesto a aceptar con un nivel de confianza que se ha definido.

Se analizaron 265 encuestas que cumplieron con todas las características, de estás 214 corresponden a usuarios femeninos y 51 a masculinos, como se muestra en la Gráfica No.1.

Gráfica No. 1 Encuestados por Género, ICSa-2018

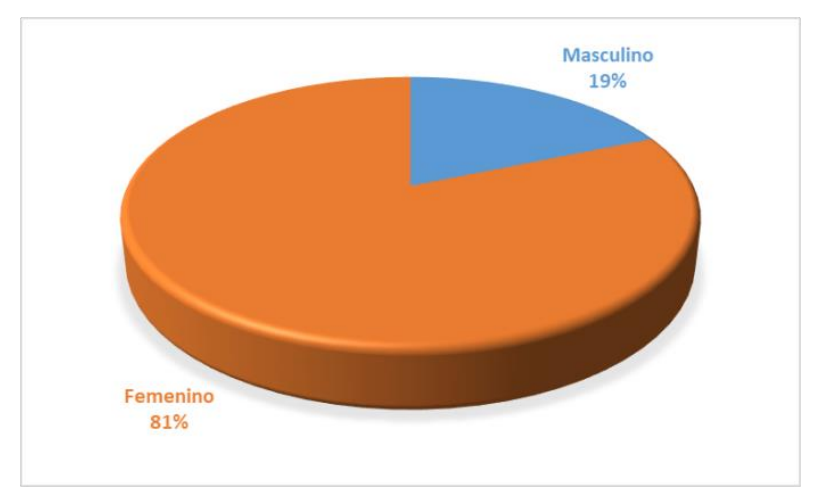

Fuente: N=265 Aplicación del Test Inteligencia Emocional.

Dentro de las variables demográficas se identificó la edad de los participantes, como se muestra en la siguiente Gráfica No. 2 Edad de los participantes.

Gráfica No. 2 Edad de los participantes, ICSa-2018

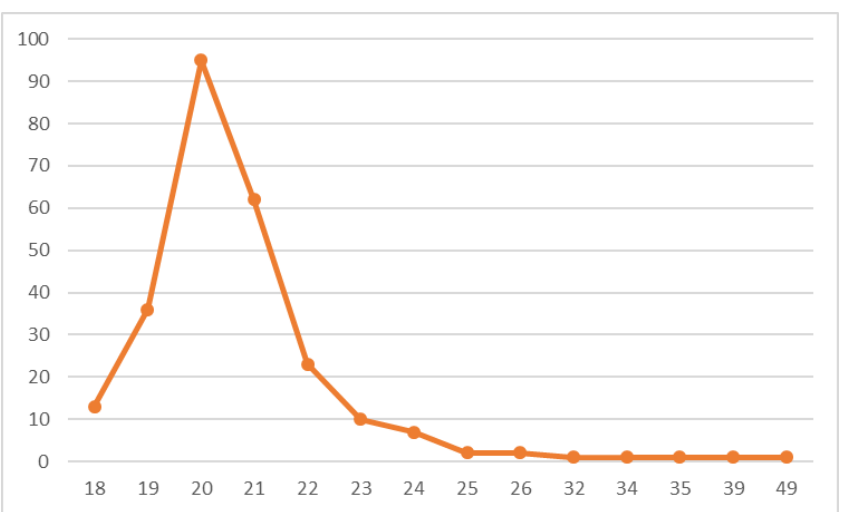

Fuente: N=265 Aplicación del Test Inteligencia Emocional.

De la gráfica identificó que los participantes tienen entre 18 y 49 años el 54\%; de 18 a 20; de 21 a 22 años el 32\%; de 23 a 24 años el 7\% y que tienen más de 25 años el 7\% del total.

Con respecto al Test de Inteligencia Emocional de Daniel Goleman, se identificó de manera general que el grupo de 255 encuestas validas trabajadas en la base de datos de SSPS, podemos entender la propuesta teórica de Daniel Goleman respecto a la inteligencia emocional es importante tener claro el concepto de emoción. Los datos que se muestran en la siguiente grafica se identifica de forma grupal que de los 5 factores de estudio 4 se encuentran el nivel medio de del diagnóstico de IE.

Tabla No. 1 Resultado de Test de Inteligencia Emocional, ICSa-2018

\begin{tabular}{|c|c|c|c|c|}
\hline \multirow{5}{*}{ FACTOR } & \multirow{5}{*}{$\begin{array}{c}\text { (SP) } \\
\text { SUMA } \\
\text { PARCIAL }\end{array}$} & \multirow{5}{*}{$\begin{array}{c}\% \\
\text { (SP X } 100 \\
\text { entre 36) }\end{array}$} & \multicolumn{2}{|c|}{ DIAGNÓSTICO } \\
\hline & & & $\%$ & NIVEL \\
\hline & & & Hasta 65 & Bajo \\
\hline & & & $66-82$ & Medio \\
\hline & & & $83-99$ & Alto \\
\hline (I) Autoconciencia & 26 & 72 & \multicolumn{2}{|c|}{ Medio } \\
\hline (II) Autocontrol & 24 & 67 & \multicolumn{2}{|c|}{ Medio } \\
\hline (III) Aprovechamiento emocional & 30 & 83 & \multicolumn{2}{|c|}{ Alto } \\
\hline (IV) Empatía & 28 & 78 & \multicolumn{2}{|c|}{ Medio } \\
\hline (V) Habilidad social & 26 & 72 & \multicolumn{2}{|c|}{ Medio } \\
\hline Total de Items & 134 & & & \\
\hline
\end{tabular}

Fuente: $\mathbf{N}=265$ Aplicación del Test Inteligencia

Como se muestra en la tabla No. 1 los resultados de todos los participantes se puede observar que se encuentran con una estabilidad emocional Media lo que se convierte en una posibilidad de trabajar con ellos para desarrollar su potencial humano.

Cabe recalcar que cada dimensión comprende varias competencias las cuales determinan la habilidad que tiene un individuo para potenciar la inteligencia emocional en los distintos aspectos de la vida cotidiana.

Ahora, los resultados obtenidos favorecen de muchas maneras a los estudiantes de la Licenciatura de Enfermería, puesto que una autoestima media o positiva aporta en la mejora de la salud física, psicológica y social y, por ende, eleva la calidad de vida, manifestándose en el desarrollo de una personalidad más plena y una percepción más satisfactoria de la vida; aumenta la capacidad de afrontar y superar las dificultades personales, pudiéndose enfrentar los problemas con actitud de confianza personal, además de fomentar la capacidad de adquirir y cumplir con los compromisos.

Por tanto, se deben aprovechar los resultados obtenidos para la implementación de estrategias que promuevan el fortalecimiento y mejora de la autoestima en los estudiantes, para con ello favorecer las capacidades que provienen como consecuencia al tener una autoestima elevada, tales como: la autovaloración, la autocrítica, la autoevaluación, la disposición a la responsabilidad propia, la asunción de decisiones propias. 
Finalmente, se deben realizar terapias psicológicas en aquellos estudiantes que obtuvieron un nivel de autoestima media y baja; por estar en riesgo de incrementarla y con ello, generar problemas de índole social, psicológica y física. De igual modo, el personal docente debe estar preparado para identificar oportunamente las características propias de la persona con baja autoestima; siendo la tendencia a la generalización, el pensamiento dicotómico o absolutista, filtrado negativo, autoacusaciones, personalización, reacción emocional poco racional, entre otros.

\section{Conclusión}

En el presente estudio se concluye que la mayoría de los estudiantes de la Licenciatura de Enfermería del Instituto de Ciencias de la Salud, presentan un nivel de Inteligencia Emocional Media, que favorece con ello que los estudiantes logren una personalidad más plena, una percepción más satisfactoria de la vida, y como estudiante, además de contar con una gran posibilidad de poder desarrollar sus potencialidades para llegar a ser un profesional competente y de calidad.

\section{Referencias}

[1] M. López, La inteligencia emocional y las estrategias de aprendizaje como predictores del rendimiento académico en estudiantes (Tesis de Maestría), Lima: Universidad Nacional Mayor de San Marcos, 208.

[2] R. Bisquerra Alzina, «GROP. Grupo de Recerca en Orientación Psicopedagogica,» 11 Noviembre 2018. [En línea]. Available: www. rafaelbisquera.com/es/inteligencia-emocional/inteligenciaemocionalsegun-salovey-mayer.html.

[3] P. Salovey, L. Stroud, A. Woolery y E. Epel, «Perceived emotional intelligence, stress reactivity, and symptom reports: Further explorations using the trait meta-mood scale» Psychology and Health, pp. 611-627, 2002.

[4] J. Ciarrochi, F. Deane y S. Anderson, «Emotional inteligence moderates the relationship between stress and mental health» Personality and Indivifual Differences, pp. 197-209, 2002.

[5] Extremera N, Fernández P. El papel de la inteligencia emocional en el alumnado: evidencias empíricas. Revista Electrónica de Investigación Educativa, 2004; 6(2): 1-14

[6] Villacorta E. Inteligencia emocional y rendimiento académico en estudiantes de Medicina Humana de la Universidad Nacional de la Amazonia Peruana. [Tesis doctoral]. Lima: Universidad Nacional Mayor de San Marcos; 2010.

[7]. Loly A, López E, Atalaya M. La autoestima y los valores organizacionales en estudiantes universitarios y no universitarios de Lima. Rev. Investigación Psicologica. 2002; 5(1): 141-155

[8] Centeno C. Influencia del Programa Explorando mis emociones en la inteligencia emocional de estudiantes del sexto grado de primaria de un Centro Educativo en Huancayo. [Tesis de pregrado]. Huancayo: Universidad Peruana Los Andes; 2007.

[9]. Castillo R. Análisis comparativo de los niveles de la inteligencia emocional en los alumnos del quinto año de secundaria con padres separados y no separados del Colegio Nacional Mixto Cartavio. [Tesis de pregrado]. Trujillo: Universidad Cesar Vallejo; 2005.
[10] Javier L, Iparraguirre M. Inteligencia emocional en estudiantes de la facultad de Ciencias de la salud de la Universidad Peruana Los Andes. Rev. Innovación 2012; (13): 121-128.

[11] Howard Garner, Las Cinco Mentes del Futuro, Barcelona, Editorial Paidós, 2007, p. 17.

[12] Manuel Muñoz Heras, Inteligencia Emocional y Pensamiento Positivo, España, Libro Hobby Club S.A., 2008, p. 27.

[13] Daniel, Goleman, Emociones Destructivas Cómo Entenderlas y Superarlas, Barcelona, Editorial Kairós, 2003, p. 210

[14] Daniel, Goleman, Inteligencia Emocional, Barcelona, Editorial Kairós, 2008 , p. 434.

[15] Mireya Vivas, Domingo Gallego, Educar las Emociones, Editorial Dykinson, Madrid, 2006, p.19

[16] Eva Adan, Jaume Cela, Emociones y educación: qué son y cómo intervenir desde la escuela, Editorial Laboratorio Educativo, España, 2007, p. 97. 